\title{
Olfactory and Taste Dysfunction in Patients with Asymptomatic and Mildly Symptomatic COVID-19 in Korea
}

\author{
Jun Yeon Won, $\mathrm{MD}, \mathrm{PhD}^{1,2}$, Yoon Heo, $\mathrm{MD}^{1}$, Tae Suk Kim, $\mathrm{MD}^{3}$, \\ Choong-Hyo Kim, $\mathrm{MD}, \mathrm{PhD}^{4}$, and Woo Hyun Lee, $\mathrm{MD}^{1}$ \\ ${ }^{1}$ Department of Otolaryngology, Kangwon National University Hospital, Chuncheon; and \\ ${ }^{2}$ Department of Otolaryngology, ${ }^{3}$ Internal Medicine, and ${ }^{4}$ Neurosurgery, Kangwon National University School of Medicine, \\ Chuncheon, Korea
}

Background and Objectives: The association between chemosensory dysfunction (CSD) and coronavirus disease 2019 (COVID-19) remains unclear. The aim of the present study was to determine the incidence of olfactory and taste dysfunction in asymptomatic and mildly symptomatic patients with COVID-19 and to evaluate the symptoms associated with CSD in patients with COVID-19.

Materials and Method: On March 9, 2020, 309 patients with asymptomatic or mildly symptomatic COVID-19 confirmed by real-time polymerase chain reaction (RT-PCR) were admitted to the No. 7 Community Treatment Center in Korea. An internetbased survey about symptomatology was administered to these patients, with responses obtained from 244 (79.0\%). Subjects who completed the survey were enrolled in this study and were categorized into either a CSD group or a normal chemosensory group based on the presence or absence of CSD, respectively.

Results: General symptoms, including fever, myalgia, and chills, were most common (29.1\%), followed by upper respiratory tract infection (URI) symptoms (20.9\%), CSD (20.5\%), and nasal symptoms (13.5\%). In patients with CSD ( $\mathrm{n}=50), 10(4.1 \%)$ reported no other symptoms. After adjustment for age, sex, past medical history, and other symptoms, general symptoms [odds ratio $(\mathrm{OR}), 3.63$; confidence interval $(\mathrm{CI}), 1.70-7.76]$ and nasal symptoms (OR, 7.00; CI, 2.61-18.80) were significantly associated with CSD.

Conclusion: The incidence of CSD was relatively high (20.5\%) in asymptomatic and mildly symptomatic patients with COVID-19. General symptoms were independent risk factors of CSD, suggesting a sensorineural mechanism for the observed olfactory and taste dysfunction.

KEY WORDS: COVID-19 $\cdot$ Smell $\cdot$ Taste $\cdot$ Asymptomatic $\cdot$ Mildly symptomatic.

\section{INTRODUCTION}

Coronavirus disease 2019 (COVID-19) caused by infection with the severe acute respiratory syndrome coronavirus 2 (SARS-CoV-2) was first reported in Wuhan, China in December of 2019. Since then, the disease has spread worldwide. Due to its rapid transmission and high mortality rates, countries all over the globe are devoting significant medical resources to the prevention and treatment of COVID-19.
A definitive diagnosis of COVID-19 can be made using a real-time polymerase chain reaction (RT-PCR) assay performed on respiratory samples. ${ }^{1)}$ Unfortunately, there is limited availability of the diagnostic test, creating barriers to testing for asymptomatic and mildly symptomatic patients who may be disease carriers. It is imperative to identify the distinctive clinical features of COVID-19 to guide strategic testing approaches for the early diagnosis and prevention of disease.

Received: September 25, 2020 / Revised: October 18, 2020 / Accepted: October 19, 2020

Address for correspondence: Woo Hyun Lee, MD, Department of Otolaryngology, Kangwon National University Hospital, 156 Baengnyeong-ro, Chuncheon 24289, Korea

Tel: +82-33-258-9109, Fax:+82-33-258-9486, E-mail: sniper212@hanmail.net 
Several recent studies have reported high prevalence of hyposmia (loss of smell) and hypogeusia (loss of taste) in COVID-19 patients. Since SARS-CoV2 is believed to have neuroinvasive potential, ${ }^{2)}$ these clinical manifestations may have important implications for early and accurate diagnosis of COVID-19. In this study, we aimed to evaluate the incidence of olfactory and taste dysfunction in asymptomatic and mildly symptomatic patients with COVID-19 and to evaluate the symptoms associated with chemosensory dysfunction (CSD) in patients with COVID-19.

\section{MATERIALS AND METHODS}

\section{Subjects}

After the first patient with COVID-19 was reported in Korea in January of 2020, over 5,000 patients with this disease were identified over the next 6 weeks. In response to this explosive increase in patients, the Korean government implemented an isolation system called 'Community Treatment Centers' (CTCs) for asymptomatic and mildly symptomatic patients diagnosed with COVID-19 using RT-PCR. CTCs were designed to facilitate the effective distribution of Korean medical resources by quarantining asymptomatic or mildly symptomatic patients in a group setting with individual lodging spaces under the supervision of medical staff. These facilities successfully helped to prevent the spread of disease in the community and provided medical care and hospital transfer to deteriorating patients. By March of 2020, a total of 12 CTCs were operating around the city of Daegu, where the disease's growth rate was most evident. This study was conducted in No. 7 CTC, one of the largest CTCs in Korea."

According to the 'Patient classification guidelines' from the Korean Centers for Disease Control and Prevention, 309 patients with asymptomatic or mildly symptomatic COVID-19 were admitted to No. 7 CTC on March 9. Asymptomatic were defined as patients who satisfy all of the following conditions: 1) a positive COVID-19 test; 2) alert mental status; 3) no underlying diseases, including diabetes mellitus, chronic kidney disease, chronic lung disease, chronic cardiovascular disease, malignancy, immunosuppressive drug therapy, or HIV; 4) age $<50$ years; 5) nonsmoker; and 6) a body temperature of $<37.5^{\circ} \mathrm{C}$ without antipyretic use. Mildly symptomatic patients were defined as meeting more than one of the following criteria in addition to a positive COVID-19 test and an alert mental status: 1) age $<50$ years; 2) more than one underlying disease; and 3) a body temperature of $<38^{\circ} \mathrm{C}$ with antipyretic use. Patients whose body temperatures exceeded $38^{\circ} \mathrm{C}$ even after antipyretic use or patients with difficulty breathing were classified as severely symptomatic and were excluded from CTC admission.

We administered an internet-based google survey (Google questionnaire; Google LLC, Mountain View, CA) to the 309 asymptomatic to mildly symptomatic patients admitted to No. 7 CTC on March 9 and received replies from 244 (79.0\%) of them. The survey queried patients about their COVID-19 symptomatology using the following statement: 'Choose symptoms you felt when your COVID-19 infection was first suspected.' Patients could choose from the following symptoms: general symptoms (i.e., fever, chills, and/or myalgia); upper respiratory tract infection (URI) symptoms (i.e., sore throat, cough, and/or sputum); nasal symptoms (i.e., nasal obstruction and/or rhinorrhea); CSD (i.e., loss of sense of smell and/or taste); chest discomfort, and diarrhea. The questionnaire also asked patients about their past medical histories, including a history of diabetes mellitus, hypertension, allergic disease, lung disease, or liver disease. The 244 patients who completed the questionnaire were included in the study and were classified into either the CSD group or the normal chemosensory (NCS) group based on the presence or absence, respectively, of hyposmia and/or hypogeusia.

\section{Statistical analysis}

Independent t-tests were performed for evaluation of age differences between the CSD and NCS groups. The chisquared test or Fisher's exact test were used for comparisons of categorical variables between the two groups. The associations between reported symptoms and the odds of CSD were evaluated using odds ratios with $95 \%$ confidence intervals obtained from logistic regression analysis. The following three logistic models were built: model 1, with no adjustments; model 2, which was adjusted for age (continuous), gender (male or female), and past medical history (diabetes mellitus, hypertension, allergic disease, lung disease, or liver disease); and model 3 (multivariate model), which was further adjusted for all other symptoms. All statistical analyses were performed using SPSS (version 18, SPSS Inc., Chicago, IL, USA), and a p-value of $<0.05$ was considered statistically significant. 


\section{Ethics statement}

The ethics committee of our hospital approved the use of the data. The need for written informed consent was waived by the institutional review board.

\section{RESULTS}

\section{Basic characteristics and clinical manifestations of COVID-19 in asymptomatic to mildly symptomatic patients}

The mean age of study participants was $36.0 \pm 15.2$ years, with a male-to-female ratio of 91:153. In the 244 included subjects, general symptoms were most commonly reported (29.1\%), followed by URI symptoms (20.9\%), CSD (20.5\%), and nasal symptoms (13.5\%). Seventeen (7.0\%) patients reported diarrhea, and $8(3.3 \%)$ patients reported chest discomfort. In addition, 42 (17.2\%) patients reported no symptoms (Fig. 1). Of the 50 patients with CSD, 8 (16.0\%) subjects reported hyposmia only, 7 (14.0\%) subjects reported hypogeusia only, and 35 (70.0\%) subjects reported both hyposmia and hypogeusia. Moreover, $10(20.0 \%)$ patients with CSD reported no other symptoms.

\section{Associated symptoms in the chemosensory dysfunction group}

Based on the presence or absence of CSD, 50 (20.5\%) and 194 (79.5\%) subjects were classified into either the CSD group or the NCS group, respectively, with comparable ages and gender ratios between the two groups. The reported prevalence of chest discomfort and diarrhea did not significantly differ between the two groups. General symptoms ( $\mathrm{p}<0.001$ ), URI symptoms ( $\mathrm{p}=0.003$ ), and nasal symptoms ( $\mathrm{p}<0.001$ ) were more prevalent in the CSD group than in the NCS group. There were no significant differences between the past medical histories of patients in the two groups (Table 1).

In logistic model 1 , the presence of general symptoms $(\mathrm{OR}=4.47 ; \mathrm{p}<0.001)$, URI symptoms $(\mathrm{OR}=2.74 ; \mathrm{p}=0.004)$, and nasal symptoms $(\mathrm{OR}=7.88 ; \mathrm{p}<0.001)$ were significantly associated with the odds of CSD. The association between these symptom types and CSD remained significant after adjustment for age, gender, and past medical history in model 2. Finally, after adjustment for age, gender, past medical history, and other reported symptoms in model 3 , the presence of general symptoms $(\mathrm{OR}=3.63 ; \mathrm{p}=0.001)$ and nasal symptoms $(\mathrm{OR}=7.00 ; \mathrm{p}<0.001)$ were determined to be independent risk factors for the odds of CSD (Table 2).

\section{DISCUSSION}

Although the COVID-19 pandemic is ongoing, the pathogenesis and clinical manifestations of this disease are still

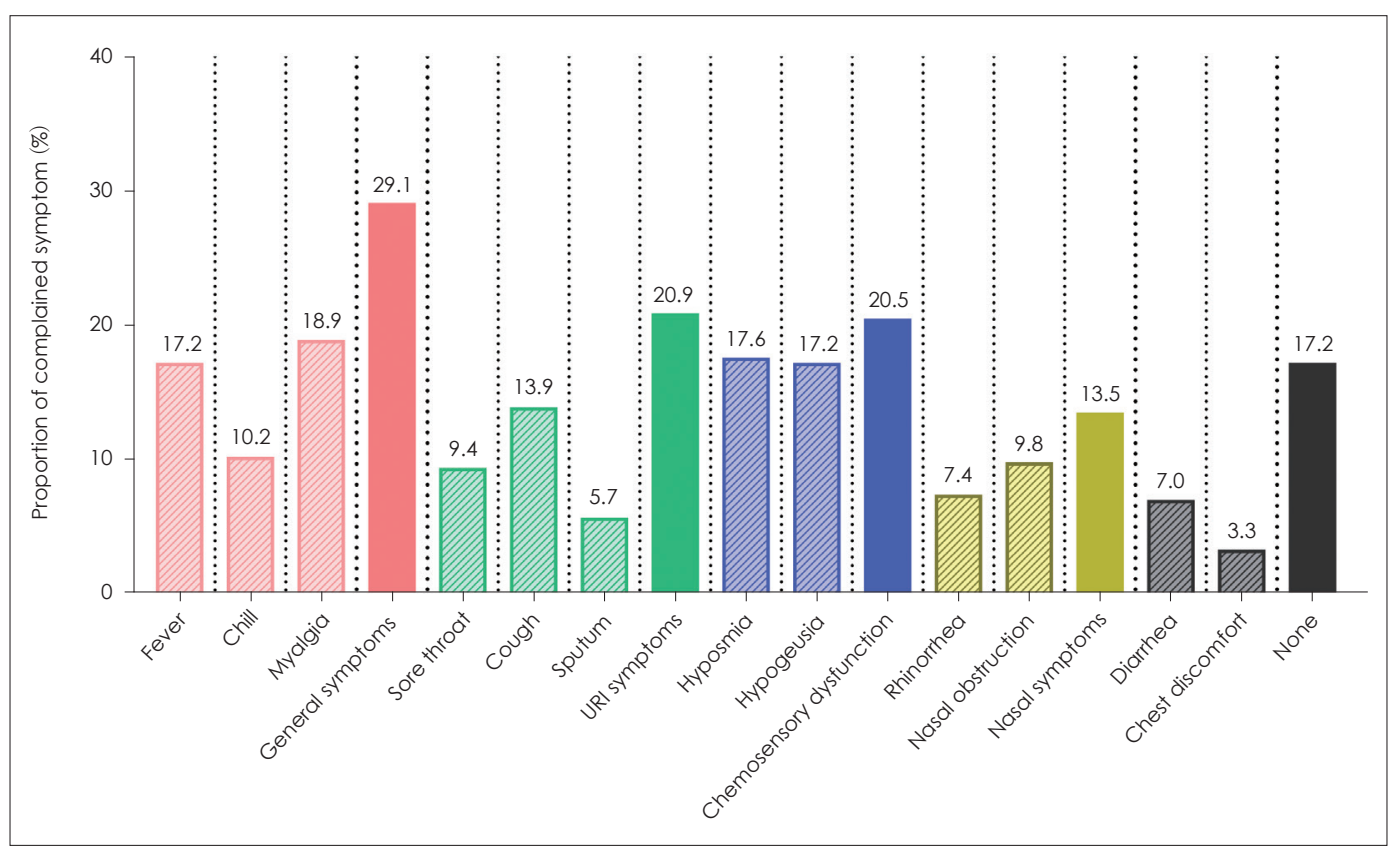

Fig. 1. Incidence of reported symptoms in study patients. General symptoms were most commonly reported, followed by upper respiratory tract infection symptoms, chemosensory dysfunction, and nasal symptoms. 
Table 1. General characteristics of the chemosensory dysfunction and normal chemosensory groups in asymptomatic and mildly symptomatic patients with coronavirus 2019 (COVID-19)

\begin{tabular}{|c|c|c|c|c|}
\hline Characteristic & $\begin{array}{c}\text { All } \\
(n=244)\end{array}$ & $\begin{array}{l}\text { Normal chemosensory } \\
\text { group }(n=194)\end{array}$ & $\begin{array}{c}\text { Chemosensory dysfunction } \\
\text { group }(n=50)\end{array}$ & p-value \\
\hline \multicolumn{5}{|l|}{ Gender, n (\%) } \\
\hline Male & $91(37.3)$ & $76(39.2)$ & $15(30.0)$ & 0.232 \\
\hline Female & $153(62.7)$ & $118(60.8)$ & $35(70.0)$ & \\
\hline Age (years), mean \pm SD & $36.0 \pm 15.2$ & $35.8 \pm 15.4$ & $37.0 \pm 14.3$ & 0.632 \\
\hline \multicolumn{5}{|l|}{ General symptoms, n (\%) } \\
\hline No & $173(70.9)$ & $151(77.8)$ & $22(44.0)$ & $<0.001$ \\
\hline Yes & $71(29.1)$ & $43(22.2)$ & $28(56.0)$ & \\
\hline \multicolumn{5}{|l|}{ URI symptoms, n (\%) } \\
\hline No & $193(79.1)$ & $161(83.0)$ & $32(64.0)$ & 0.003 \\
\hline Yes & $51(20.9)$ & $33(17.0)$ & $18(36.0)$ & \\
\hline \multicolumn{5}{|l|}{ Nasal symptoms, n (\%) } \\
\hline No & $211(86.5)$ & $180(92.8)$ & $31(62.0)$ & $<0.001$ \\
\hline Yes & $33(13.5)$ & $14(7.2)$ & $19(38.0)$ & \\
\hline \multicolumn{5}{|l|}{ Chest discomfort, n (\%) } \\
\hline No & $236(96.7)$ & $189(97.4)$ & $47(94.0)$ & 0.211 \\
\hline Yes & $8(3.3)$ & $5(2.6)$ & $3(6.0)$ & \\
\hline \multicolumn{5}{|l|}{ Diarrhea, n (\%) } \\
\hline No & $227(93.0)$ & $183(94.3)$ & $44(88.0)$ & 0.126 \\
\hline Yes & $17(7.0)$ & $11(5.7)$ & $6(12.0)$ & \\
\hline \multicolumn{5}{|l|}{ Diabetes mellitus, n (\%) } \\
\hline No & $241(98.8)$ & $191(98.5)$ & $50(100.0)$ & 1.000 \\
\hline Yes & $3(1.2)$ & $3(1.5)$ & $0(0.0)$ & \\
\hline \multicolumn{5}{|l|}{ Hypertension, n (\%) } \\
\hline No & $230(94.3)$ & $180(92.8)$ & $50(100.0)$ & 0.080 \\
\hline Yes & $14(5.7)$ & $14(7.2)$ & $0(0.0)$ & \\
\hline \multicolumn{5}{|l|}{ Heart disease, n (\%) } \\
\hline No & $243(99.6)$ & $193(99.5)$ & $50(100.0)$ & 1.000 \\
\hline Yes & $1(0.4)$ & $1(0.5)$ & $0(0.0)$ & \\
\hline \multicolumn{5}{|l|}{ Allergic disease, n (\%) } \\
\hline No & $230(94.3)$ & $183(94.3)$ & $47(94.0)$ & 1.000 \\
\hline Yes & $14(5.7)$ & $11(5.7)$ & $3(6.0)$ & \\
\hline \multicolumn{5}{|l|}{ Lung disease, n (\%) } \\
\hline No & $242(99.2)$ & $192(99.0)$ & $50(100.0)$ & 1.000 \\
\hline Yes & $2(0.8)$ & $2(1.0)$ & $0(0.0)$ & \\
\hline \multicolumn{5}{|l|}{ Liver disease, n (\%) } \\
\hline No & $243(99.6)$ & $193(99.5)$ & $50(100.0)$ & 1.000 \\
\hline Yes & $1(0.4)$ & $1(0.5)$ & $0(0.0)$ & \\
\hline
\end{tabular}

URI: upper respiratory tract infection

not fully understand. In order to construct an effective prevention strategy, it is necessary to detect patients in the early stages of COVID-19 and to block the contact of carriers with their communities. It is therefore of vital importance to understand the clinical manifestations of this disease in asymptomatic and mildly symptomatic patients. This study was conducted to determine the clinical manifestations of
COVID-19 in asymptomatic and mildly symptomatic patients, with a specific focus on CSD. In this study, we found a $20.5 \%$ prevalence of CSD in asymptomatic and mildly symptomatic patients with COVID-19, with $20.0 \%$ of these patients having no other symptoms.

Previously reported incidences of CSD in patients with COVID-19 varied according to the study. A Chinese study 
Table 2. Multivariable logistic regression model predicting chemosensory dysfunction in asymptomatic or mildly symptomatic patients with coronavirus disease 2019 (COVID-19)

\begin{tabular}{|c|c|c|c|c|c|c|c|}
\hline & \multirow{2}{*}{ Cases/at risk } & \multicolumn{2}{|c|}{ Model 1* } & \multicolumn{2}{|l|}{ Model $2^{\dagger}$} & \multicolumn{2}{|c|}{ Model $3^{+}$} \\
\hline & & OR $(95 \% \mathrm{Cl})$ & p-value & OR $(95 \% \mathrm{Cl})$ & $\mathrm{p}$-value & OR $(95 \% \mathrm{Cl})$ & $\mathrm{p}$-value \\
\hline General symptoms & $28 / 244$ & $4.47(2.32-8.59)$ & $<0.001$ & $4.16(2.13-8.14)$ & $<0.001$ & $3.63(1.70-7.76)$ & 0.001 \\
\hline URI symptoms & $18 / 244$ & $2.74(1.38-5.46)$ & 0.004 & $2.73(1.34-5.58)$ & 0.006 & $0.77(0.30-1.99)$ & 0.585 \\
\hline Nasal symptoms & $19 / 244$ & $7.88(3.58-17.34)$ & $<0.001$ & $7.45(3.33-16.67)$ & $<0.001$ & $7.00(2.61-18.80)$ & $<0.001$ \\
\hline Chest discomfort & $3 / 244$ & $2.41(0.58-10.46)$ & 0.239 & $1.95(0.44-8.62)$ & 0.380 & $0.84(0.17-4.09)$ & 0.828 \\
\hline Diarrhea & $6 / 244$ & $2.27(0.80-6.48)$ & 0.125 & $2.25(0.76-6.68)$ & 0.144 & $0.98(0.29-3.34)$ & 0.975 \\
\hline
\end{tabular}

*Model 1: crude model, no adjustment, † Model 2: adjusted for age, gender, and past medical histories, $¥$ Model 3: adjusted for age, gender, past medical histories, and other symptom. OR: odds ratio, URI: upper respiratory tract infection

published at the beginning of the epidemic reported that the CSD incidence in patients with COVID-19 ranged from 5.1 to $5.6 \%{ }^{4)}$ In Italian studies, the CSD incidence in COVID-19 patients ranged from 19.4 to $33 \%,{ }^{56)}$ and, in a hospital study from the United States, the incidence of CSD was reported to be $70 \%{ }^{7)}$ Considering that our study was conducted in asymptomatic and mildly symptomatic patients who are believed to have a relatively low level of viral activity or to be at an early stage of disease, the observed $20.5 \%$ incidence of CSD may demonstrate the clinical value of this symptom for the early diagnosis of disease. While previous studies were limited in their ability to measure CSD because of patient condition or because they were performed in older people who may have had preexisting CSD, our study was conducted in a relatively young patient population (mean age: $36.0 \pm 15.2$ years) with only mild symptoms, which may ensure the reliability of our results. Therefore, if patients present with newly developed CSD, even if it is their only symptom, clinicians should consider performing RT-PCR assays for identification of COVID-19 during this pandemic.

There are known to be two types of olfactory loss in patients, sensorineural and conductive. The olfactory dysfunction observed in COVID-19 patients seems to be associated with the sensorineural type. In transgenic mice, it has previously been shown that coronaviruses administered intranasally can enter the brain through the olfactory nerve. ${ }^{899}$ In addition, previous study hypothesized that the olfactory loss observed in COVID-19 patients may have an underlying sensorineural mechanism through SARSCoV-2's interaction with the olfactory epithelium. ${ }^{\text {.) }}$ If the observed olfactory loss in COVID-19 had an underlying conductive mechanism, it would be always expected that nasal symptoms that block the spread of odorants, including nasal obstruction and rhinorrhea, would also be present. However, our study showed that general symptoms, including fever, myalgia, and chills, were independent risk factors for the development of CSD, which provides further evidence of sensorineural olfactory dysfunction in patients with COVID-19. These results suggest that olfactory dysfunction can develop regardless of the presence of nasal symptoms in patients with COVID-19.

This study had several limitations. First, participant recall bias was possible in this survey-based study. Second, selection bias may have occurred in this internet-based study because patients with poor progress or poor computer literacy may have been excluded. Third, we did not perform endoscopic nor radiologic exam of sinus to the participant. Therefore, conductive olfactory loss was not completely excluded.

\section{Acknowledgments}

We would like to express our sincerest gratitude to Kangwon National University Hospitals medical staff who devoted their effort to patient's care in No. 7 CTC.

This research received no specific grant from any funding agency in the public, commercial, or not-for-profit sectors.

\section{REFERENCES}

1) Bénézit F, Le Turnier P, Declerck C, Paillé C, Revest M, Dubée V, et al. Utility of hyposmia and hypogeusia for the diagnosis of COVID-19. The Lancet Infectious Diseases;2020.

2) Li YC, Bai WZ, Hashikawa T. The neuroinvasive potential of SARSCoV2 may play a role in the respiratory failure of COVID-19 patients. Journal of Medical Virology 2020;92(6):552-5.

3) Park PG, Kim CH, Heo Y, Kim TS, Park CW, Kim CH. Out-of-hospital cohort treatment of coronavirus disease 2019 patients with mild symptoms in Korea: an experience from a single community treatment center. J Korean Med Sci 2020;35(13):e140.

4) Mao L, Jin H, Wang M, Hu Y, Chen S, He Q, et al. Neurologic manifestations of hospitalized patients with coronavirus disease 2019 in Wuhan, China. JAMA Neurol 2020;77(6):683-90.

5) Vaira LA, Salzano G, Deiana G, De Riu G. Anosmia and ageusia: common findings in COVID-19 patients. Laryngoscope 2020;130 (7):1787.

6) Giacomelli A, Pezzati L, Conti F, Bernacchia D, Siano M, Oreni L, et al. Self-reported olfactory and taste disorders in patients with se- 
vere acute respiratory coronavirus 2 infection: a cross-sectional study. Clinical Infectious Diseases;2020.

7) Yan CH, Faraji F, Prajapati DP, Boone CE, DeConde AS. Association of chemosensory dysfunction and COVID-19 in patients presenting with influenza-like symptoms. Int Forum Allergy Rhinol 2020;10(7):806-13

8) Netland J, Meyerholz DK, Moore S, Cassell M, Perlman S. Severe acute respiratory syndrome coronavirus infection causes neuronal death in the absence of encephalitis in mice transgenic for human ACE2. Journal of Virology 2008;82(15):7264-75.

9) Li K, Wohlford-Lenane C, Perlman S, Zhao J, Jewell AK, Reznikov LR, et al. Middle East respiratory syndrome coronavirus causes multiple organ damage and lethal disease in mice transgenic for human dipeptidyl peptidase 4. The Journal of Infectious Diseases 2016; 213(5):712-22. 